\title{
MICROORGANISMOS FUNCIONALES EN SUELOS CON Y SIN REVEGETALIZACIÓN EN EL MUNICIPIO DE VILLA DE LEYVA, BOYACÁ
}

\author{
Functional microorganisms in soill with and without revegetation \\ in the municipality of Villa de Leyva-Boyaca
}

\author{
Mayra Eleonora Beltrán Pineda', Zulma Edelmira Rocha Gil², Andrea Angélica Bernal Figueroa ${ }^{3}$ \\ \& Luz Adriana Pita Morales ${ }^{4}$
}

Beltrán Pineda, M.E., Rocha Gil, Z.E., Bernal Figueroa, A.A. \& Pita Morales, L.A. (2017). Microorganismos funcionales en suelos con y sin revegetalización en el municipio de Villa de Leyva, Boyacá. Colombia Forestal, 20(2), 158-170.

Recepción: 4 de mayo 2016

\section{Resumen}

Las iniciativas en restauración ecológica que incluyen técnicas de revegetalización, son procesos complejos que pueden ser afectados por muchas variables bióticas y abióticas. Dentro de estas últimas, las poblaciones de microorganismos juegan un rol fundamental en la liberación de nutrientes y por tanto en el establecimiento de especies vegetales en las áreas a restaurar. El estudio buscó evaluar la densidad de las comunidades microbianas relacionadas con los ciclos de nutrientes del carbono, fósforo y nitrógeno conocidas como grupos funcionales presentes en un suelo revegetalizado y otro sin revegetalizar en el municipio de Vila de Leyva. Los métodos empleados en esta evaluación fueron recuento en placa estándar utilizando medios de cultivo selectivos, también se realizó análisis de correlación entre grupos para evidenciar posibles interacciones de tipo ecológico. En general las poblaciones de todos los grupos funcionales de microorganismos están presentes en las dos zonas evaluadas. Solo se encontraron diferencias significativas para los recuentos de las bacterias solubilizadoras de fosfato y las poblaciones de microorganismos proteolíticos $(p \leq 0,05)$.
Aprobación: 22 de mayo de 2017

Se encontraron algunas correlaciones estadísticamente significativas entre unos grupos microbianos que podrían indicar procesos de cometabolismo y competencia entre poblaciones microbianas del suelo, los cuales favorecen el equilibrio ecológico del mismo.

Palabras clave: microorganismos del suelo, restauración ecológica, revegetalización.

\begin{abstract}
Ecological restoration initiatives that include revegetation techniques are complex processes that can be affected by many biotic and abiotic variables. Among the latter, populations of microorganisms play a fundamental role in the release of nutrients and therefore in the establishment of plant species in the areas to be restored. The study sought to evaluate the density of microbial communities related to the nutrient cycles of carbon, phosphorus, and nitrogen known as functional groups present in a revegetated soil and another zone without intervention in the municipality of Villa de Leyva. Methods used in this evaluation were standard plate counts using selective culture media, also a correlation analysis
\end{abstract}

\footnotetext{
Grupo de Investigación Gestión Ambiental. Universidad de Boyacá.Tunja, Colombia. mebeltran@uniboyaca.edu.co. Autor de correspondencia. Grupo de Investigación Gestión Ambiental. Universidad de Boyacá. Tunja, Colombia zerocha@uniboyaca.edu.co Grupo de Investigación Gestión Ambiental. Universidad de Boyacá. Tunja, Colombia aabernal@uniboyaca.edu.co Grupo de Investigación Gestión Ambiental. Universidad de Boyacá. Tunja, Colombia. luzpita@uniboyaca.edu.co
} 
was also performed between groups to evidence possible ecological interactions. In general populations of all functional groups of microorganisms are present in the two zones evaluated. Only significant differences were found for counts of phosphate solubilizing bacteria and proteolytic microorganism populations $(p \leq 0.05)$. Some statistically significant correlations were found between some microbial groups that could indicate processes of cometabolism and competition among microbial populations of the soil, which favors the ecological balance of the same.

Keywords: soil microorganisms, ecological restoration, revegetation.

\section{INTRODUCCIÓN}

Los ecosistemas brindan importantes servicios para el ser humano como la regulación del clima, purificación del agua, descontaminación del aire, control de inundaciones, recreación, paisaje, entre otros. Sin embargo, la constante presión antrópica sobre los hábitats naturales ha provocado que estos disminuyan la capacidad de suministrar los servicios al mismo nivel que en el pasado, poniendo en riesgo algunas actividades económicas y la salud humana (Fernández et al., 2010). Las actuales tasas de fragmentación y pérdida de hábitats han llevado a que varias especies se encuentren en peligro de extinción y muchos ecosistemas se encuentren degradados (Huxel \& Hastings, 1999). Por lo tanto, su recuperación es una estrategia de vital importancia para la conservación biológica.

En este mismo sentido en los últimos años en Colombia se han desarrollado varias iniciativas de recuperación de ecosistemas enfocadas a diferentes factores como: generar ciclaje de nutrientes, aumentar biomasa, promover la formación de suelos, entre otros; esto con el fin de activar las cadenas tróficas. Según (Bashan et al., 2015), la reforestación de áreas erosionadas es esencial para revertir los impactos provocados, debido a que la carencia de vegetación conlleva a problemas ambientales y genera riesgos para la salud de la población.

La restauración ecológica es una actividad intencional que busca, mediante la aceleración de los procesos sucesionales naturales, iniciar o acelerar la recuperación de un ecosistema nativo con respecto a su salud, integridad y sustentabilidad
(Fernández et al., 2010). La revegetalización hace parte de los procesos de restauración y se define como el fenómeno por el cual las plantas colonizan un área de la cual ha sido removida su cobertura vegetal original por efecto de un disturbio (Vargas, 2007). Esta técnica ha sido reportada como una alternativa para disminuir la erosión del suelo y su degradación (Hou et al., 2002) y para restaurar la integridad ecológica de ecosistemas (Cheng \& An, 2015).

Se conoce que el éxito del proceso de revegetalización puede verse influenciado por la cantidad de actividad microbiana en los suelos que han sido afectados y por la cantidad de vínculos entre plantas y microorganismos en el área (Archer \& Pyke, 1991). Los grupos funcionales de microorganismos involucrados en diferentes etapas de los ciclos de nutrientes son parte integral de los procesos de revegetalización y pueden ser usados como indicadores de la calidad del suelo o como una herramienta biotecnológica disponible para reducir su erosión en general (Bashan et al., 2015).

El municipio de Villa de Leyva (Boyacá) ha presentado históricamente una vocación ganadera y de cultivos extensivos, generando suelos con alta susceptibilidad a la erosión y desertificación (Alcaldía de Villa de Leyva, 2012). A partir de esta situación se han gestionado algunas iniciativas para revegetalizar ciertas zonas degradas como instrumento para la protección de suelos. Este estudio buscó evaluar la densidad de las comunidades microbianas relacionadas con los ciclos de nutrientes del carbono, fósforo y nitrógeno presentes en un suelo revegetalizado y otro sin intervención en 
una zona localizada en la vereda Ritoque Alto, del municipio de Villa de Leyva. Los métodos empleados en esta evaluación permiten establecer posibles interacciones ecológicas que se presenten en los suelos de la zona.

\section{MATERIALES Y MÉTODOS}

\section{Área de estudio y toma de muestras}

La zona de estudio se ubicó en la vereda Ritoque Alto, del municipio de Villa de Leyva en la provincia Ricaurte-Boyacá, se encuentra localizada sobre las coordenadas de latitud: $5^{\circ} 37^{\prime} 16.2^{\prime \prime} \mathrm{N}$ y longitud: $73^{\circ} 31^{\prime} 6.4^{\prime \prime} \mathrm{O}$ y a $2.262 \mathrm{~m}$ de altitud; los suelos presentan alta susceptibilidad a la erosión, aridización y desertificación. Para la obtención de las muestras de suelo se seleccionaron dos zonas contrastantes, una revegetalizada con cuatro especies nativas de la zona (Baccharis macrantha, Baccharis latifolia, Crotalaria micans y Myrsinea coriacea) y otra sin ningún tipo de intervención, a la cual se definió como zona sin revegetalizar. Para la recolección de las muestras de suelo en cada terreno se realizó el método zigzagueo con una distancia entre puntos de cinco metros (Usda, 1999), utilizando el barreno en los vértices a una profundidad de $30 \mathrm{~cm}$ y teniendo en cuenta las condiciones de terreno en donde fuera posible la recolección de muestra Por lo anterior, se tomaron muestras adicionales lo más cercanas a cada punto de muestreo hasta completar la cantidad de muestra necesaria para los análisis. Las muestras fueron conservadas a $4{ }^{\circ} \mathrm{C}$ para su posterior análisis fisicoquímico y microbiológico.

\section{Análisis fisicoquímicos de los suelos}

Los parámetros fisicoquímicos de los suelos en estudio se determinaron de acuerdo a técnicas estandarizadas (Horwitz, 2000) en el laboratorio de diagnóstico en suelos y aguas de la Universidad Pedagógica y Tecnológica de Colombia. Se utilizó para textura el método de Bouyoucos, (Bouyoucos, 1962); pH la relación 1:1 suelo: agua; porcentaje de materia orgánica según Walkey-Black, (Walkley \& Black, 1934); fósforo disponible por el método de Bray II y colorimetría (Bray \& Kurtz, 1945); AI mediante acidez intercambiable: $\mathrm{KCl} 1 \mathrm{~N}$; $\mathrm{Ca}, \mathrm{Mg}$, $\mathrm{K}, \mathrm{Na}, \mathrm{Fe}, \mathrm{Mn}, \mathrm{Cu}, \mathrm{Zn}$ por extracción con absorción atómica, $\mathrm{B}$ por el método de agua caliente y S usando fosfato monocálcico.

\section{Recuentos de grupos funcionales de microorganismos}

Los recuentos de estos grupos funcionales se realizaron mediante diluciones seriadas y siembra en medio selectivo por triplicado para cada muestra determinando el Log de UFC.g ${ }^{-1}$ de suelo. Para los microorganismos celulolíticos se utilizó el medio de cultivo Agar CMC (Carboximetil celulosa) según Samira et al. (2011). Este medio contiene $\mathrm{K}_{2} \mathrm{HPO}_{4} 1.0$ g.l $\mathrm{I}^{-1}, \mathrm{MgSO}_{4} .7 \mathrm{H}_{2} \mathrm{O} 0.5 \mathrm{g.l}^{-1}$;

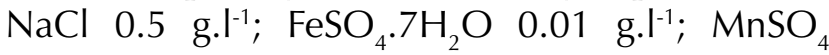

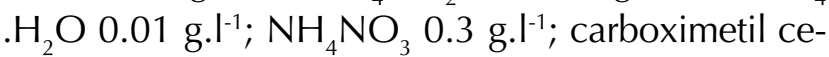

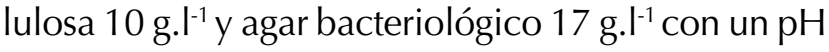
final de 7.0. Las cajas se incubaron por 7 días a $28^{\circ} \mathrm{C}$ y luego se realizó el recuento de las colonias que muestran halos de hidrólisis utilizando Rojo Congo al $1 \%$ y $\mathrm{NaCl} 1 \mathrm{M}$ como agentes reveladores según la metodología propuesta por Samira et al. (2011).

Para los microorganismos amilolíticos se utilizó el medio de cultivo Agar Almidón según Rodríguez et al. (2006); este medio contiene triptona $10 \mathrm{g.l}^{-1}$;

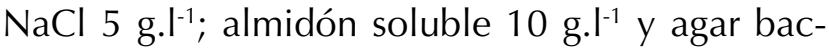

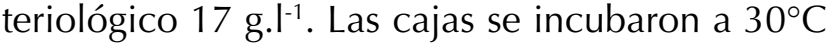
por 72 horas para las bacterias y por 7 días para los hongos; luego se realizó el recuento de las colonias que mostraron halos de hidrólisis utilizando como agente revelador Lugol al 0.05\% según Rodríguez et al. (2006).

Para las poblaciones de proteolíticos se utilizó el medio de cultivo Agar Leche según Bizuye et al. (2014). Este medio contiene leche descremada en

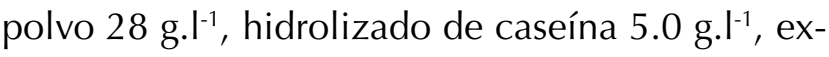

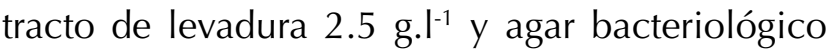


15 g..$^{-1}$ a un $\mathrm{pH}$ final de $7.0 \pm 0.2$. Las cajas se incubaron a $37^{\circ} \mathrm{C}$ por 48 horas; luego se realizó el recuento de las colonias que mostraron halos de hidrólisis que se observaron de color transparente según lo propuesto por Bizuye et al. (2014).

Para el grupo de los solubilizadores de fosfato se utilizó el medio de cultivo NBRIP (National Botanical Research Institute Phosphate growth médium), según Nautiyal (1999), este medio con-

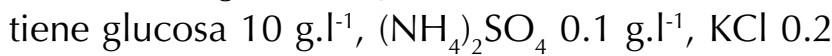

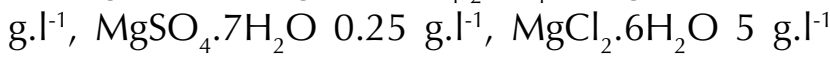
y agar bacteriológico $14 \mathrm{g.t}^{-1}$ con un $\mathrm{pH}$ final de 7 y enriquecido con fosfato tricálcico $\mathrm{Ca}_{3} \mathrm{PO}_{4}$ al $0.5 \%$ como fuente de fosfato inorgánico e insoluble. Las cajas se incubaron a $28^{\circ} \mathrm{C}$ durante 14 días y al final del periodo de incubación se realizó el recuento de microorganismos solubilizadores de fosfato tricálcico que pueden seleccionarse por la aparición de halos claros alrededor de las UFC, según Nautiyal (1999). Finalmente, para las poblaciones de microorganismos fijadores libres de nitrógeno, se utilizó el medio de cultivo Agar Ashby sólido según Wen Xu et al. (2012); este medio con-

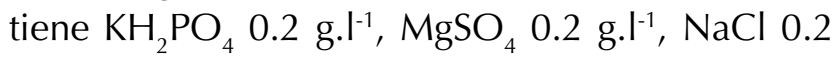

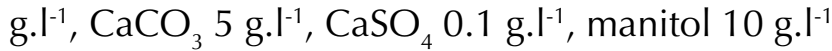
y agar bacteriológico $15 \mathrm{g.l}^{-1} \mathrm{a} \mathrm{pH} \mathrm{7.0.} \mathrm{Las} \mathrm{cajas}$ fueron incubadas a $28^{\circ} \mathrm{C}$ durante 7 días hasta la observación de colonias traslucidas y mucoides según Wen Xu et al. (2012).

\section{Análisis estadístico}

Para realizar el análisis comparativo de las poblaciones microbianas en cada una de las zonas estudiadas (revegetalizada y sin revegetalizar) se utilizó la prueba t-Student. Adicionalmente, se realizaron pruebas de correlación de Pearson entre los recuentos de los grupos funcionales presentes en cada una de las zonas para conocer las posibles interacciones y relaciones ecológicas. El análisis estadístico se realizó con ayuda del programa Minitab® Release 14 Statistical Software y los gráficos se realizaron mediante el software SIGMAPLOT versión 10.0.

\section{RESULTADOS}

\section{Análisis fisicoquímico de los suelos}

En el análisis fisicoquímico de los suelos evaluados se observa que tanto en los suelos revegetalizados como sin revegetalizar se presentan texturas franco arenosas y $\mathrm{pH}$ ácidos (tabla 1). La zona revegetalizada muestra CICE y contenidos de $\mathrm{MO}$, P y K más bajos que los reportados en la zona sin revegetalización. Por su parte, los contenidos de Ca y $\mathrm{S}$ indican valores más altos en la zona sin revegetalizar, pero en cuanto a los demás elementos

Tabla 1. Análisis fisicoquímico de suelos revegetalizados y sin revegetalizar.

\begin{tabular}{|c|c|c|c|c|c|c|c|c|c|c|}
\hline \multirow{2}{*}{ Tipo de suelo } & \multirow{2}{*}{ Textura } & \multirow{2}{*}{$\mathbf{p H}$} & \multirow{2}{*}{$\%$ MO } & \multirow{2}{*}{$\begin{array}{l}\text { P(ppm) } \\
\text { Bray II }\end{array}$} & \multicolumn{6}{|c|}{ meq/100g de suelo-cmol*. $\mathrm{Kg}^{-1}$} \\
\hline & & & & & $\mathrm{Al}$ & $\mathrm{Ca}$ & $\mathrm{Mg}$ & K & $\mathrm{Na}$ & CICE \\
\hline \multirow{3}{*}{ Revegetalizado } & Franco arenoso & 4.4 & 12.65 & 5.46 & $\begin{array}{l}5.3 \\
\text { Ppm }\end{array}$ & 0.92 & 0.65 & 0.25 & 0.16 & 11.38 \\
\hline & S & $\mathrm{Fe}$ & Mn & $\mathrm{Cu}$ & $\mathrm{Zn}$ & B & \multicolumn{4}{|c|}{ Densidad real $\mathrm{g} / \mathrm{cm}^{3}$} \\
\hline & 18.5 & 125 & 17.48 & 0.17 & 7.81 & 0.36 & \multicolumn{4}{|c|}{2.13} \\
\hline \multirow{3}{*}{ Sin revegetalizar } & Franco arenoso & 4.4 & 15.07 & 7.95 & $\begin{array}{l}7.4 \\
\text { Ppm }\end{array}$ & 0.71 & 0.53 & 0.39 & 0.15 & 13.18 \\
\hline & S & $\mathrm{Fe}$ & $\mathrm{Mn}$ & $\mathrm{Cu}$ & $\mathrm{Zn}$ & B & \multicolumn{4}{|c|}{ Densidad real $\mathrm{g} / \mathrm{cm}^{3}$} \\
\hline & 22.0 & 122 & 4.62 & 0.1 & 6.36 & 0.32 & \multicolumn{4}{|c|}{2.50} \\
\hline
\end{tabular}


la zona revegetalizada muestra concentraciones mayores de Fe, Mn, Cu, Zn y B. Con relación a la densidad real, estadísticamente no hay diferencias entre los dos terrenos evaluados.

\section{Recuentos de grupos funcionales de microorganismos}

\section{Microorganismos celulolíticos, amilolíticos y proteolíticos}

El recuento de microorganismos celulolíticos para la zona revegetalizada fue de $4.01 \mathrm{Log}^{\mathrm{UFC}} . \mathrm{g}^{-1} \mathrm{de}$ suelo y en la zona sin revegetalización fue de 4.02 Log UFC. g $^{-1}$ de suelo (figura 1). En este caso no se evidencian diferencias significativas en el recuento de este tipo de poblaciones $(p>0,05)$, a pesar de que según el análisis fisicoquímico de los suelos los contenidos de materia orgánica difieren en las dos zonas analizadas, siendo superior para el suelo sin revegetalización. En este estudio el recuento de los microorganismos amilolíticos en la

Recuento de microorganismos celulolíticos-amilolíticos y proteolíticos

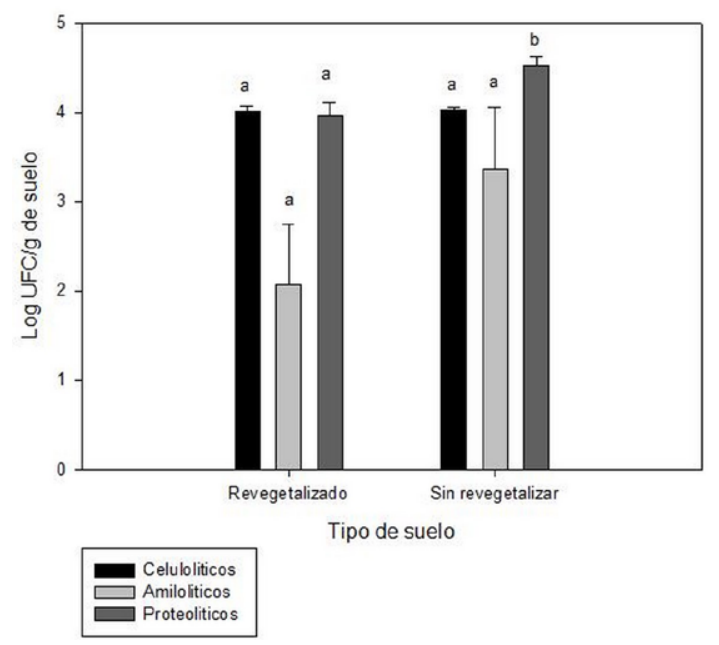

Figura 1. Recuento de microorganismos celulolíticos, amilolíticos y proteolíticos en suelos con y sin revegetalización en el municipio de Villa de Leyva. Las barras corresponden a la media con su respectivo error estándar. Letras iguales no difieren significativamente según la prueba t-student al $5 \%$. zona revegetalizada fue de 2.07 Log UFC.g ${ }^{-1}$ de suelo y en la zona sin revegetalizar fue de 2.36 Log UFC.g-g de suelo (figura 1). Al igual que en las poblaciones celulolíticas, no se evidencia una diferencia significativa entre zonas $(p>0,05)$. En el caso de los microorganismos proteolíticos el recuento fue de $3.97 \mathrm{Log}$ UFC.g $^{-1}$ de suelo para la zona revegetalizada y $4.52 \mathrm{Log}_{\mathrm{UFC} . \mathrm{g}^{-1}}$ en la zona sin revegetalizar (figura 1), en este caso sí se presentaron diferencias estadísticamente significativas entre las zonas en estudio $(p \leq 0,05)$.

\section{Microorganismos solubilizadores de fosfatos} Con respecto a los microorganismos funcionales del ciclo de fósforo evaluados, se evidencian diferencias estadísticamente significativas para los dos sitios en estudio $(p \leq 0,05)$, el recuento de microorganismos solubilizadores de fosfato para la zona

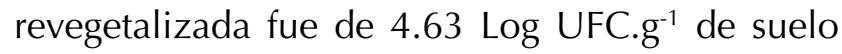
y en la zona sin revegetalización fue de $4.85 \mathrm{Log}$ UFC.g ${ }^{-1}$ de suelo (figura 2).

Recuento de microorganismos solubilizadores de fosfato y fijadores libres de nitrógeno

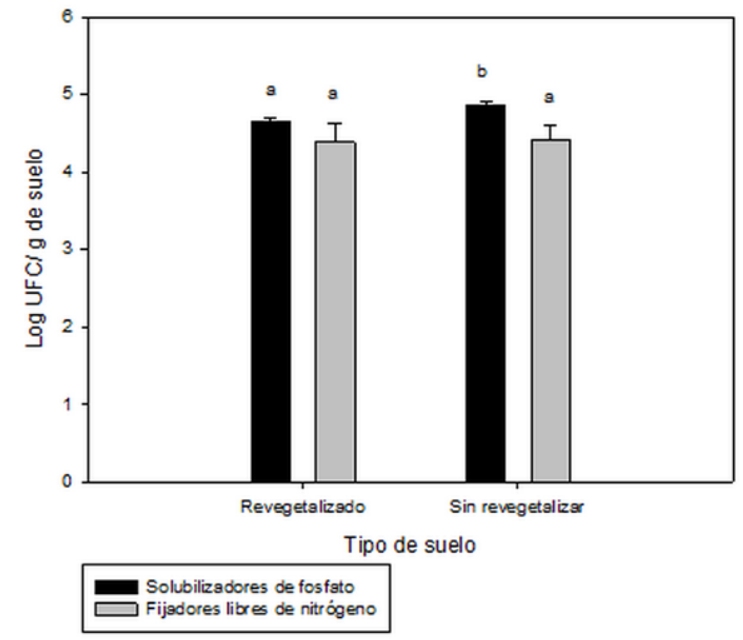

Figura 2. Recuento de microorganismos solubilizadores de fosfato y fijadores libres de nitrógeno en suelos con y sin revegetalización en el municipio de Villa de Leyva. Las barras corresponden a la media con su respectivo error estándar. Letras iguales no difieren significativamente según la prueba t-student al 5\%. 
Microorganismos libres fijadores de nitrógeno

El recuento de microorganismos fijadores de nitrógeno para la zona revegetalizada fue de $4.38 \mathrm{Log}$ UFC. ${ }^{-1}$ de suelo y en la zona sin revegetalización fue de 4.42 Log UFC.g-1 de suelo (figura 2). No se presentaron diferencias estadísticamente significativas entre las zonas estudiadas ( $p>0.05)$, al igual que en algunos de los grupos funcionales también evaluados.

\section{Correlaciones entre los grupos funcionales de} microorganismos para las dos zonas analizadas Para la zona revegetalizada se encontró una correlación positiva estadísticamente significativa entre las poblaciones de bacterias proteolíticas y las solubilizadoras de fosfato (tabla 2). Para la zona sin revegetalización no se encontraron correlaciones estadísticamente significativas entre la mayoría de los grupos funcionales de microorganismos analizados, a excepción de la correlación negativa encontrada entre las poblaciones de solubilizadores de fosfato y los fijadores libres de nitrógeno que resulto ser significativa.

\section{DISCUSIÓN}

\section{Análisis fisicoquímico de suelos}

Los procesos de fragmentación que se han generado por la actividad antrópica producen la alteración en varios procesos ecológicos, como el ciclaje de nutrientes, los cuales afectan el ecosistema a nivel global y por lo tanto a los organismos vivos asociados. En cuanto a parámetros como el pH y la conductividad, Mummey et al. (2002) indican que estos influencian el proceso de revegetalización. Sin embargo, en las dos zonas estudiadas se presentó crecimiento vegetal a pesar de la condición acida de los suelos, razón por la cual en el estudio este factor no se considera determinante para el establecimiento de las especies vegetales. La zona revegetalizada muestra CICE y contenidos de $\mathrm{MO}$, P y K más bajos que los reportados en la zona sin revegetalización, esta situación es opuesta a lo reportado por Cao et al. (2008), quienes indican que después del establecimiento de la planta pionera C. microphylla en un proceso de revegetalización

Tabla. 2. Correlación lineal entre los grupos funcionales de microorganismos del ciclo de $\mathrm{C}, \mathrm{N}$ y $\mathrm{P}$ en una zona con y sin revegetalización en el municipio de Villa de Leyva.

\begin{tabular}{|c|c|c|c|c|c|c|c|c|c|}
\hline \multicolumn{6}{|c|}{ SUELO REVEGETALIZADO } & \multicolumn{4}{|c|}{ SUELO SIN REVEGETALIZAR } \\
\hline & SF & C & A & $\mathbf{p}$ & & SF & $\mathbf{C}$ & A & $\mathbf{p}$ \\
\hline C & $\begin{array}{l}\mathrm{r}:-0.388 \\
\mathrm{p}: 0.448\end{array}$ & & & & C & $\begin{array}{l}\mathrm{r}:-0.630 \\
\mathrm{p}: 0.180\end{array}$ & & & \\
\hline A & $\begin{array}{l}\mathrm{r}: 0.764 \\
\mathrm{p}: 0.077\end{array}$ & $\begin{array}{l}\mathrm{r}: 0.589 \\
\mathrm{p}: 0.219\end{array}$ & & & A & $\begin{array}{l}\mathrm{r}:-0.255 \\
\mathrm{p}: 0.626\end{array}$ & $\begin{array}{l}\mathrm{r}:-0.454 \\
\mathrm{p}: 0.366\end{array}$ & & \\
\hline$P$ & $\begin{array}{l}\text { r: } 0.879 \\
\text { p: } 0.021\end{array}$ & $\begin{array}{l}\text { r: }-0.356 \\
\text { p: } 0.489\end{array}$ & $\begin{array}{l}\text { r: } 0.454 \\
\text { p: } 0.366\end{array}$ & & $P$ & $\begin{array}{l}\mathrm{r}: 0.132 \\
\mathrm{p}: 0.804\end{array}$ & $\begin{array}{l}r:-0.275 \\
\text { p: } 0.598\end{array}$ & $\begin{array}{l}\text { r: } 0.498 \\
\text { p: } 0.314\end{array}$ & \\
\hline FN & $\begin{array}{l}\text { r:-0.333 } \\
\text { p:0.519 }\end{array}$ & $\begin{array}{l}\text { r: } 0.175 \\
\text { p:0.740 }\end{array}$ & $\begin{array}{l}\text { r:-0.318 } \\
\text { p:0.540 }\end{array}$ & $\begin{array}{l}r:-0.271 \\
\mathrm{p}: 0.603\end{array}$ & $\mathrm{FN}$ & $\begin{array}{l}\mathrm{r}:-0.905 \\
\mathrm{p}: 0.013\end{array}$ & $\begin{array}{c}r:-0.410 \\
\text { p:0.419 }\end{array}$ & $\begin{array}{l}\mathrm{r}:-0.087 \\
\mathrm{p}: 0.870\end{array}$ & $\begin{array}{l}r:-0.517 \\
\mathrm{p}: 0.294\end{array}$ \\
\hline
\end{tabular}

C: celulolíticos; A: amilolíticos, P: proteolíticos; SF: solubilizadores de fosfato; FN: fijadores libres de nitrógeno. r: coeficiente de correlación de Pearson; $\mathrm{p}=$ valor $\mathrm{P}$. 
la biomasa de $\mathrm{C}$ y $\mathrm{N}$ aumenta, la fijación de nitrógeno y su liberación por la descomposición de la hojarasca incrementa el contenido de este nutriente en los suelos, con el aumento de la edad de la plantación el desarrollo de hierbas anuales y perennes forman un componente importante de la productividad primaria neta y su rápido crecimiento y muerte provee un influjo importante de $\mathrm{C}, \mathrm{N}$ y otros nutrientes al sistema. Así mismo, el desarrollo de raíces incrementa los contenidos de $\mathrm{MO}$ y provee una fuente de carbono para el metabolismo de las poblaciones microbianas de la rizósfera (Matsumoto et al., 2005).

Por otra parte, se conoce que con el progreso de la sucesión vegetal la fijación de carbono por medio de la fotosíntesis y la subsecuente transferencia del $\mathrm{C}$ al suelo a través de la hojarasca contribuyen a la acumulación de C orgánico en el suelo y mejoran la calidad del mismo (Zhang et al., 2012; Cheng \& An, 2015). Con respecto a la fracción nitrogenada de la $\mathrm{MO}$ en suelos revegetalizados, Hu et al. (2009), Fu et al. (2010) y Cheng \& An (2015) encontraron que la concentración de $\mathrm{N}$ en los suelos se incrementa con el aumento del tiempo de revegetalización. Por su parte, Wen et al. (2005) manifiestan que los niveles de $\mathrm{P}$ disponible aumentan con el tiempo de revegetalización; y Li et al. (2007) indicaron que la revegetalización mejora las condiciones ambientales para la colonización y el establecimiento de nuevas especies vegetales. Sin embargo, el análisis fisicoquímico del suelo de la zona intervenida no refleja esta situación.

Los contenidos de Ca y $\mathrm{S}$ presentaron valores más altos en la zona sin revegetalizar, pero en cuanto a los demás elementos la zona revegetalizada muestra contenidos más altos de Fe, Mn, $\mathrm{Cu}, \mathrm{Zn}$ y $\mathrm{B}$. Con referencia a la densidad real no se evidencian diferencias marcadas entre los dos terrenos evaluados, esto podría explicarse desde los conocimientos generados en experiencias de restauración en donde los resultados son variables debido a que es un proceso ecológico complejo afectado simultáneamente por muchas variables bióticas y abióticas (Dangi et al., 2011).

\section{Recuentos de microorganismos funcionales en los suelos}

La importancia de las interacciones planta-microorganismos en la estructura de los ecosistemas es ampliamente reconocida y dicha asociación es esencial para el crecimiento de cualquier especie vegetal. En el análisis realizado se evidencia que la densidad de la mayoría de los grupos de microorganismos se incrementan con relación a disponibilidad de nutrientes, algunos por procesos de descomposición, otros por solubilización, como es el caso del fosfato inorgánico transformándolo a ortofosfato, que es una forma asimilable para las plantas; este tipo de adaptabilidad especifica por parte de los microorganismos hace que estas poblaciones se denominen grupos funcionales (Bashan et al., 2015).

\section{Microorganismos celulolíticos}

El alimento es parte del ecosistema microbiano y es uno de los factores más importantes que determinan la comunidad que se desarrolla sobre él, dentro del sustrato los carbohidratos desempeñan un papel determinante (Rodríguez et al., 2006). El crecimiento microbiano depende del influjo de materiales orgánicos como la celulosa, lo cual depende en gran medida de la disponibilidad de $\mathrm{C}$ en los suelos (Cao et al., 2008). En este sentido, es conocido que los microorganismos de la rizósfera son capaces de usar un amplio rango de moléculas orgánicas complejas como lignina, celulosa y otros polisacáridos, una consecuencia de dicha habilidad es que estos no dependen de los exudados radiculares para sobrevivir (Matsumoto et al., 2005).

Estos grupos funcionales han sido evaluados en otras zonas como el páramo de Rabanal en el departamento de Boyacá por Beltrán \& Lizarazo (2013), quienes encontraron hasta 4.3 Log UFC.g-1 de suelo; por otra parte, en cultivos de interés agronómico como la cebolla junca Torres \& Lizarazo (2006) reportaron $4.63 \mathrm{Log}_{\mathrm{UFC}} \mathrm{g}^{-1}$ de suelo que son valores comparables a los reportados en la zonas evaluadas. 
La abundancia de microorganismos del suelo se relaciona con la distribución espacial de los recursos pues se sabe que con la profundidad esta disminuye debido a que declina la concentración de carbono orgánico (Helgason et al., 2014). De otra parte, Cao et al. (2008) afirman que la actividad microbiana del suelo es mayor en la superficie del suelo que en las capas profundas, debido a que el suministro de carbono fresco que proviene de la biomasa vegetal y el incremento de materiales orgánicos e inorgánicos provee sustratos para una comunidad microbiana abundante y activa. En este estudio las muestras de suelo para el análisis microbiológico fueron tomadas a poca profundidad, esto podría explicar por qué los recuentos encontrados son comparables con los reportados para suelos agrícolas.

Con el progreso de la sucesión vegetal se incrementa la fijación de carbono por medio de la fotosíntesis y la subsecuente transferencia de $\mathrm{C}$ al suelo a través de la hojarasca y el ciclaje a nivel de la raíz, lo que contribuye a su acumulación (Cheng \& An, 2015). Esto haría pensar que en la zona con intervención las poblaciones de microorganismos celulolíticos serían superiores, pero en este caso esta situación no ocurre, a pesar de que las plántulas utilizadas en el proceso de restauración llevan establecidas cerca de cinco años, y en términos de presencia de microorganismos celulolíticos no se puede asegurar que el proceso de restauración ha sido progresivo y ha mejorado las propiedades químicas y microbiológicas del suelo, como lo indican Cao et al. (2008), pues las poblaciones son invariables en las dos zonas contrastantes.

\section{Microorganismos amilolíticos}

El almidón y la celulosa son las dos fuentes de carbono más distribuidas en la naturaleza. Estos polímeros están compuestos de unidades de glucosa y se requieren de diferentes sistemas enzimáticos para degradarlos (Arellano \& Olmos, 1999). Los grupos microbianos con actividad amilolítitica juegan un papel fundamental en el ciclaje de nutrientes a nivel de suelo, pues actúan sobre el almidón gracias a la producción de enzimas extracelulares denominadas amilasas, liberando glucosa que es un sustrato más fácilmente asimilable por el resto de las poblaciones heterótrofas de microorganismos (Beltrán \& Lizarazo, 2013).

La adecuada proporción de materia orgánica facilita el establecimiento de estas poblaciones en las dos zonas en estudio. Se conoce que el humus en suelos es una fuente importante de minerales y nutrientes orgánicos como el almidón para las poblaciones de microorganismos nativos (Cheng \& An, 2015). Pero, en esta investigación, este grupo funcional fue el que presentó menos abundancia para las dos zonas estudiadas, lo que podría relacionarse con la disponibilidad de sustratos carbonáceos fácilmente asimilables, como es la glucosa que se libera producto de la hidrólisis del almidón. Se sabe que los factores ambientales como la reducción en la disponibilidad de oxígeno y la baja temperatura pueden hacer más lentos procesos de descomposición por la disminución de la velocidad del metabolismo y la reducción de la actividad de las enzimas extracelulares como las amilasas (Helgason et al., 2014).

La fertilidad del suelo está controlada por las actividades biogeoquímicas de la microbiota que actúa como abastecedor potencial de nutrientes para las plantas, como es el caso de las poblaciones de amilolíticos (Sivila de Cary \& Angulo, 2006), estas poblaciones han sido evaluadas en otros ecosistemas perturbados como suelos afectados por incendios forestales en el páramo de Rabanal, encontrando poblaciones de hasta 4.8 Log UFC.g ${ }^{-1}$ de suelo (Beltrán \& Lizarazo, 2013), que son valores superiores a los registrados en esta investigación.

\section{Microorganismos proteolíticos}

El carbón orgánico del suelo se constituye de varios componentes que difieren en sus propiedades físico-químicas y en el grado de estabilización y tiempo de reciclado (Abakumov et al., 2013). Las proteínas son un componente fundamental de la materia orgánica del suelo, por eso el rol de las poblaciones proteolíticas es crucial. Gracias a la 
producción de enzimas proteasas se produce la liberación de compuestos menores asimilables, como los aminoácidos que hacen parte de la materia orgánica particulada y que pueden ser rápidamente accesibles para los microorganismos (Zimmermann et al., 2007).

Algunos factores que pueden afectar la actividad y tasa de crecimiento microbiana en lo suelos incluyen la disponibilidad de oxígeno y la fertilidad del mismo (Cao et al., 2008). Los microorganismos tienen el potencial de competir con las raíces de las plantas por aminoácidos libres en la solución del suelo, especialmente cuando la actividad microbiana es intensa como ocurre en la rizósfera (Matsumoto et al., 2005); esto explicaría la menor proporción de este tipo de microorganismos en la zona revegetalizada.

En lo concerniente a la zona intervenida, la proporción de estas poblaciones disminuye con respecto a su contraparte en la zona sin intervención. Es importante reconocer que reducciones en la actividad de los microorganismos del ciclo del C pueden influenciar la fertilidad del suelo, su estructura y, por lo tanto, la habilidad de los suelos para liberar nutrientes. Además, la ausencia de microorganismos puede afectar severamente el establecimiento de plántulas, el crecimiento y la sobrevivencia (Bashan et al., 2015). Estas poblaciones han sido evaluadas en suelos perturbados como en el estudio realizado por Beltrán \& Lizarazo (2013), encontrando valores de hasta 4.6 Log UFC.g ${ }^{-1}$ de suelo que son valores comparables a los obtenidos en esta investigación.

\section{Microorganismos solubilizadores de fosfato} Linares (1999) indica que los microorganismos solubilizadores hacen parte de la microflora nativa de los suelos y se encuentran en niveles de hasta 4.23 Log UFC. g $^{-1}$ de suelo. Sin embargo, los valores reportados en este estudio son superiores. La revegetalización ha sido reportada como la forma más efectiva para abatir la erosión del suelo, su degradación y como una alternativa para restaurar la integridad ecológica en ecosistemas perturbados
(Cheng \& An, 2015). Adicionalmente, es sabido que la abundancia de diversos grupos microbianos, incluidos los solubilizadores, está ligada directamente a la abundancia de plantas y actividad fisiológica de las mismas (Archer \& Pyke, 1991). En general, parece existir un efecto rizosférico sobre dichos organismos debido a que los exudados radicales y detritus vegetales proporcionan el sustrato energético para que sea posible la actividad microbiológica solubilizadora de fosfato (Valero, 2003). A pesar de esto, en la zona sin revegetalización la densidad de este grupo funcional fue superior.

El grupo funcional de los solubilizadores de fosfato está presente en varios tipos de ecosistemas tanto naturales como agroecosistemas (Beltrán, 2014). La evaluación de este tipo de poblaciones se ha realizado principalmente a nivel de agroecosistemas como suelos cultivados con cebolla junca, papa y arroz, en donde se encontraron en todos los casos valores comparables a los registrados en este estudio (Torres \& Lizarazo, 2006; Vanegas et al., 2013; Beltrán \& Lizarazo, 2013). A pesar de lo reportado por Carniero et al. (2004), quienes indicaron que los suelos agrícolas pueden presentar valores de UFC más altos que los suelos forestales y que uno de los factores que más incide sobre este parámetro es el $\mathrm{pH}$ del suelo modificado por la adición de fertilizantes, lo que también favorece al incremento de nutrientes.

Este grupo funcional de microorganismos es el que muestra la mayor abundancia para las dos zonas analizadas. Al respecto, Van der Heijden et al. (2008) reportan que los microorganismos solubilizadores pueden llegar a constituir cerca del $40 \%$ de la población cultivables en el suelo. Pero, según lo reportado por Torres \& Lizarazo (2006), las bacterias solubilizadoras de fosfato se encuentran en menor proporción que los grupos funcionales del ciclo del carbono. Sin embargo, al comparar sus resultados con los obtenidos en esta investigación concuerdan en que el grupo funcional de los solubilizadores de fosfato mantiene tamaños poblacionales similares a los del grupo de los fijadores de nitrógeno. 


\section{Microorganismos libres fijadores de nitrógeno} Las bacterias fijadoras de nitrógeno pueden favorecer el establecimiento de las plantas, aumentar su productividad e incrementar la explotación de los suelos en términos de agua y nutrientes, también aumentan la cantidad de nutrientes a nivel del foIlaje y permiten aumentar las tasas de sucesión en los sitios revegetalizados (Archer \& Pike, 1991).

En este sentido, el estudio registró que este tipo de comunidades microbianas se han logrado establecer en las dos zonas y esto es importante ya que las reducciones en la actividad de microorganismos rizosféricos fijadores de nitrógeno puede influenciar en la fertilidad de suelos, estructura y, por tanto, su capacidad de liberar nutrientes (Archer \& Pike, 1991). Los microorganismos fijadores libres de nitrógeno contribuyen al mantenimiento de la diversidad a través de su influencia en la disponibilidad de diferentes fuentes de nitrógeno tanto orgánico como inorgánico en los suelos (Van der Heijden et al., 2008).

En ecosistemas disturbados las reducciones en la biomasa vegetal pueden reducir la energía para los organismos del suelo (Archer \& Pike, 1991). La evaluación de microorganismos fijadores de nitrógeno en suelos perturbados por incendios forestales realizada por Beltrán \& Lizarazo (2013), donde indicaron poblaciones de hasta 5.4 Log UFC.g ${ }^{-1}$ de suelo, los cuales son valores superiores a los encontrados en esta investigación, lo que podría indicar que estas poblaciones se recuperan rápidamente tras algún tipo de disturbio o condiciones ambientales poco favorables.

El establecimiento y persistencia de las plantas vasculares en proyectos de restauración podrían facilitarse si se hicieran esfuerzos para introducir bacterias fijadoras de nitrógeno de vida libre en los terrenos (Archer y Pike, 1991). En esta investigación se evidencia que estas poblaciones están presentes en los suelos de manera natural y son componentes importantes del ciclo de nitrógeno en la zona. Dado que en los suelos se presenta una competencia entre las poblaciones de la rizósfera y las plantas por los compuestos de $\mathrm{C}$ y $\mathrm{N}$ disponibles, esto puede afectar a las poblaciones de bacterias fijadoras de nitrógeno y a las plantas establecidas (Matsumoto et al., 2005; Van der Heijden et al., 2008).

Correlaciones entre los grupos funcionales de microorganismos

En la zona revegetalizada se encontró una correlación positiva entre bacterias proteolíticas y las solubilizadoras de fosfato, lo que podría indicar que los grupos bacterianos están actuando sinérgicamente posibilitando mecanismos de cometabolismo (Atlas \& Bartha, 2002). Esta correlación ya ha sido encontrada en el estudio realizado por Torres \& Lizarazo (2006), quienes evaluaron diferentes grupos funcionales en suelo rizosférico y suelo total de cultivos de cebolla y papa. El resto de correlaciones entre grupos funcionales no fueron estadísticamente significativas (tabla 2).

Para la zona sin intervención se encontró una correlación negativa entre las poblaciones de los solubilizadores de fosfato y los fijadores de nitrógeno. Esta relación podría implicar un fenómeno de competencia por nutrientes o espacio entre las dos poblaciones microbianas implicadas. A diferencia de lo reportado en esta investigación, Torres \& Lizarazo (2006) evidenciaron una correlación positiva entre los grupos de solubilizadores de fosfato y los fijadores de nitrógeno en suelos de cultivos de cebolla y papa. Adicionalmente, Beltrán \& Lizarazo (2013) encontraron una correlación positiva entre esos dos grupos funcionales de microorganismos en suelos de páramo afectados por incendios forestales.

En síntesis, se conoce que en áreas en donde los procesos de revegetalización no prosperan es porque generalmente la capa superficial del suelo se ha perdido y con ella los microorganismos benéficos asociados a las plantas y consecuentemente su fertilidad (Bashan et al., 2015). Sin embargo, en esta investigación se pudo encontrar que dichos grupos funcionales evaluados hacen parte de la microflora normal del suelo, que están presentes tanto en suelos revegetalizados como en suelos sin revegetalización y que si bien la literatura indica 
que su abundancia debería ser mayor en zonas intervenidas y en proceso de restauración, debido a la gran variedad de vínculos planta-microorganismo que se establecen en la zona rizosférica, esto no siempre se cumple.

\section{CONCLUSIONES}

El estudio mostró que los grupos funcionales evaluados hacen parte de la microflora normal del suelo y están presentes en los suelos con y sin revegetalización, debido a que en las dos zonas evaluadas no se evidenciaron diferencias significativas en el recuento de microorganismos celulolíticos y amilolíticos, a pesar de que el contenido de materia orgánica difiere en las dos zonas analizadas, siendo superior para el área sin revegetalización.

De los grupos funcionales caracterizados en la investigación, los microorganismos proteolíticos y las bacterias solubilizadoras de fosfato presentaron diferencias estadísticamente significativas entre zonas de estudio. Lo anterior se asocia a los procesos naturales de sucesión en donde algunas plantas nativas se desarrollan en el terreno disturbado y promueven el establecimiento de estos grupos de microorganismos, que son los encargados de garantizar el ciclaje de nutrientes en el suelo.

En cuanto a los resultados comparativos entre zonas de estudio, el área con revegetalización registró una correlación positiva estadísticamente significativa entre las poblaciones de bacterias proteolíticas y solubilizadoras de fosfato, que podrían indicar procesos de cometabolismo y competencia entre poblaciones microbianas del suelo, los cuales favorecen el equilibrio ecológico del mismo. En la zona sin revegetalización no se encontraron correlaciones estadísticamente significativas entre la mayoría de los grupos funcionales de microorganismos analizados, a excepción de la correlación negativa registrada entre las poblaciones de solubilizadores de fosfato y los fijadores libres de nitrógeno, la cual resultó ser significativa.
Finalmente, el estudio mostró que en procesos de restauración ecológica, las técnicas de revegetalización promueven cambios en la dinámica de nutrientes en el suelo, generando el establecimiento de grupos funcionales de microorganismos en las diferentes etapas de procesos sucesionales.

\section{CONFLICTO DE INTERESES}

El autor declara no tener conflicto de intereses.

\section{CONTRIBUCIÓN POR AUTOR}

El autor único es responsable de la obra en todos los aspectos que condujeron a la elaboración de su publicación.

\section{REFERENCIAS BIBLIOGRÁFICAS}

Abakumov, E., Cajthaml, T., Brus, J. \& Frouz, J. (2013). Humus accumulation, humification and humic acid composition in soils of two post-mining chronosequences after coal mining. Journal of Soils Sediments, 13, 491- 500.

Alcaldía de Villa de Leyva. (2012). Plan de desarrollo municipal 2012-2015. Villa de Leyva: Alcaldía Municipal. $34 \mathrm{p}$.

Archer, S. \& Pyke, D. (1991). Plant-animal interactions affecting plant establishment and persistence on revegetated rangeland. Journal of Range Management, 44(8), 558-565.

Arellano, F. \& Olmos, J. (1999). Enzimas amilolíticas microbianas. Biotecnologia, 4(3), 115-121.

Atlas, R. \& Bartha, R. (2002). Ecología microbiana y Microbiología ambiental. Madrid-España: Pearson Educación S.A. 608 p.

Bashan, Y., Puente, E., Salazar, B., De-Bashan, L., Bacilio, M., Hernández, J., Leyva, L., Romero, B., Villalpando, R. \& Bethlenfalvay, G. (2015). Reforestación de tierras erosionadas en el desierto: el papel de las bacterias promotoras de crecimiento 
en plantas y la materia orgánica. Suelos Ecuatoriales, 35 (1), 70-77.

Beltrán, M. \& Lizarazo, L. (2013). Grupos funcionales de microorganismos en suelos de páramo afectados por incendios forestales. Revista de Ciencias, 17(2), 121-136.

Beltrán, M. (2014). La solubilización de fosfatos como estrategia microbiana para promover el crecimiento vegetal. Corpoica Ciencia y Tecnología Agropecuaria, 15(1), 101-113.

Bizuye, A., Sago, A., Admasu, G., Getachew, H., Kassa, P. \& Amsaya, M. (2014). Isolation, optimization and characterization of protease producing bacteria from soil and water in Gondar town, North West Ethiopia. International Journal of Bacteriology, Virology and Immunology, 1(3), 020-024.

Bouyoucos, G. (1962). Hydrometer method improved for making particle size analysis of soil. Agronomy Journal, 54, 464-465.

Bray, R. \& Kurtz, L. (1945). Determination of total, organic and available forms of phosphorus in soils. Soil Science, 59(1), 39-46.

Cao, C., Jiang, D., Teng, X., Jiang, Y., Liang, W. \& Cui, Z. (2008). Soil chemical and microbiological properties along a chronosequence of Caragana microphylla Lam. plantations in the Horqin sandy land of Northeast China. Applied soil Ecology, 40,78-85.

Carniero, R., Mendes, L., Lovato, P., Carvalhi, A. \& Vivaldi, L. (2004). Indicadores biológicos associados ao ciclo del fósforo em solos de cerrado sob plantio direto e plantio convencional. Pesquisa Agropecuaria Brasilera, 39(7), 661-669.

Cheng, M. \& An, S. (2015). Responses of soil nitrogen, phosphorous and organic matter to vegetation succession on the Loess Plateauof China. Journal Arid Land, 7(2), 216-223.

Dangi, S., Stahl, P., Wick, A. Ingram, L. \& Buyer, J. (2011). Soil microbial community recovery in reclaimed soils on a surface coal mine site. Soil Science Society of America Journal, 76, 915-924.

Fernández, I., Morales, N., Olivares, L., Salvatierra, J., Gómez, M. \& Montenegro, G. (2010). Restauración ecológica para ecosistemas nativos afectados por incendios forestales. Santiago de Chile: Gráfica Lom y Concha y Toro 25. 162 p.

Fu, X., Shao, M. \& Wei, X. (2010). Soil organic carbon and total nitrogen as affected by vegetation types in Northern Loess Plateau of China. Geoderma, 155, 31-35.

Helgason, B., Konschunh, H., Bedardrd, A. \& Vanderbygaart, A. (2014). Microbial distribution in an eroded landscape: Buried A horizons support abundant and unique communities. Agriculture Ecosystems and Environment, 196, 94-102.

Horwitz, W. (2000). Official Methods of Analysis of AOAC International. Gaithersburg, MA: AOAC International. $771 \mathrm{p}$.

Hou, F., Xiao, J. \& Nan, Z. (2002). Eco-restoration of abandoned farmland in the Loess Plateau. China. Chinese Journal of Applied Ecology, 13(8), 923-929.

Hu, C., Fu, B. \& Jin, T. (2009). Effects of vegetation restoration on soil microbial biomass carbon and nitrogen in hilly areas of Loess Plateau. Chinese Journal of Applied Ecology, 20(1), 45-50.

Huxel, G. \& Hastings, A. (1999). Habitat loss, fragmentation and restoration. Restoration Ecology, 7, 309-315.

Li, X., Kong, D. \& Tan, H. (2007). Changes in soil and vegetation following stabilization of dunes in the southeastern fringe of the Tengger Desert, China. Plant and Soil, 300, 221-231.

Linares, N. (1999). Efecto de las bacterias solubilizadoras de fósforo en la producción del cultivo de arroz (Trabajo de pregrado, Facultad de Agronomía). Bogotá: Universidad Nacional de Colombia. 70 p.

Matsumoto, L., Martines, A., Avanzi, M., Albino, U., Brazil, C., Saridakis, D., Rampazo, L., Zangaro, W. \& Andrade, G. (2005). Interactions among functional groups in the cycling of carbon, nitrogen and phosphorus in the rhizosphere of three successional species of tropical woody trees. Applied soil Ecology, 28, 57-65.

Minitab Inc. (2003). MINITAB Statistical Software, Release 14 for Windows, State College, Pennsylvania.

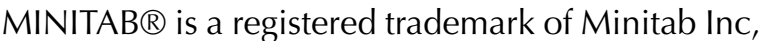

Mummey, D., Stahl, P. \& Buyer, J. (2002). Soil microbiological and physiochemical properties 20 years 
after surface mine reclamation: Comparative spatial analysis of reclaimed and undisturbed ecosystems. Soil Biology Biochemistry, 34, 1717-1725.

Nautiyal, C. (1999). An efficient microbiological growth medium for screening phosphate-solubilizing microorganisms. FEMS Microbiology Letters, 170, 265-270.

Rodríguez, Z., Boucourt, R., Rodríguez, J., Albelo, N., Nuñez, O. \& Herrera, F. (2006). Aislamiento y selección de microorganismos con capacidad de degradar el almidón. Revista Cubana de Ciencia Agrícola, 40(3), 349-354.

Samira, M., Mohammad, R. \& Gholamreza, G. (2011). Carboxymethyl-cellulase and filter-paperase activity of new strains isolated form Persian Gulf. Microbiology Journal, 1(1), 8-16.

Sivila de Cary, R. \& Angulo, W. (2006). Efecto del descanso agrícola sobre la microbiota del suelo (Patarani-Altiplano Central boliviano). Ecología en Bolivia, 41(3), 103-115.

Systat Software Inc. (SSI). (2003 -2013). SigmaPlot Versión 10.0. [software de computadora en CD-ROM]. United States of America. Recuperado de: http:/ www.sigmaplot.co.uk/products/sigmaplot/sigmaplot-details.php

Torres, M. \& Lizarazo, L. (2006). Evaluación de grupos funcionales del ciclo del C, N y P y actividad de la fosfatasa ácida en dos suelos agrícolas del departamento de Boyacá. Agronomía Colombiana, 24(2), 317-325.

United States Department of Agriculture (Usda). (1999). Guía para la evaluación de la calidad y salud del suelo. Estados Unidos: Usda. 82 p.

Valero, N. (2003). Potencial biofertilizante de bacterias diazotróficas y solubilizadoras de fosfato asociadas al cultivo de arroz (Oriza sativa) (Trabajo de Maestría, Ciencias-Microbiología). Bogotá: Universidad Nacional de Colombia. 92 p.
Van der Heijden, M., Bardgett, R., Van Straalen, N. (2008). The unseen majority: soil microbes as drivers of plant diversity and productivity in terrestrial ecosystems. Ecology Letters, 11, 296-310.

Vanegas, J., Landazabal, J., Melgarejo, L., Beltrán, M., Uribe, D. (2013). Structural and functional characterization of the microbial communities associated with the upland and irrigated rice rhizospheres in a neotropical Colombian savannah. European Journal of Soil Biology, 55, 1-8.

Vargas, O. (Ed.). (2007). Guía metodológica para la restauración ecológica del bosque altoandino. Bogotá: Convenio Interinstitucional Acueducto de Bogotá, Jardín Botánico, Secretaría Distrital de Ambiente, Grupo de Restauración Ecológica Universidad Nacional de Colombia. 194 p.

Walkley, A. \& Black, I. (1934). An examination of Degtjareff method for determining soil organic matter and a proposed modification of the chromic acid titration method. Soil Science, 37,29-38.

Wen X, Zhi Yang, Ch., Jiao Chena, Y., Min Chena, L., Zhu Zhanga, D., Meia, L., Tao shia, Y. \& Bo zhanga, H. (2012). Changes in non-symbiotic nitrogen-fixing bacteria inhabiting rhizosphere soils of an invasive plant Ageratina adenophora. Applied Soil Ecology, 54, 32-38.

Wen, Z., Jiao, F., Liu, B. (2005). Natural vegetation restoration and soil nutrient dynamic of abandoned farmlands in forest-steppe zone on Loess Plateau. Chinese Journal of Applied ecology, 16(11), 2025-2029.

Zhang, C., Liu, G. \& Xue S. (2012). Rhizosphere soil microbial properties on abandoned croplands in the Loess Plateau, China during vegetation succession. European Journal of Soil Biology, 50,127-136.

Zimmermann, M., Leifeld, J. \& Fuhrer, J. (2007). Quantifying soil organic fractions by infrared-spectroscopy. Soil Biology \& Biochemistry, 39,224-231.

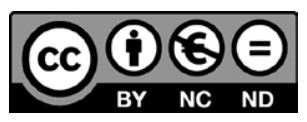

